\title{
From sudden quench to adiabatic dynamics in the attractive Hubbard model
}

\author{
Giacomo Mazza ${ }^{1,2, *}$ \\ ${ }^{1}$ Centre de Physique Théorique, École Polytechnique, \\ CNRS, Université Paris-Saclay, 91128 Palaiseau, France \\ ${ }^{2}$ Collège de France, 11 place Marcelin Berthelot, 75005 Paris, France
}

\begin{abstract}
We study the crossover between the sudden quench limit and the adiabatic dynamics of superconducting states in the attractive Hubbard model. We focus on the dynamics induced by the change of the attractive interaction during a finite ramp time. The ramp time is varied in order to track the evolution of the dynamical phase diagram from the sudden quench to the equilibrium limit. Two different dynamical regimes are realized for quenches towards weak and strong coupling interactions. At weak coupling the dynamics depends only on the energy injected into the system, whereas a dynamics retaining memory of the initial state takes place at strong coupling. We show that this is related to a sharp transition between a weak and a strong coupling quench dynamical regime, which defines the boundaries beyond which a dynamics independent from the initial state is recovered. Comparing the dynamics in the superconducting and non-superconducting phases we argue that this is due to the lack of an adiabatic connection to the equilibrium ground state for non-equilibrium superconducting states in the strong coupling quench regime.
\end{abstract}

\section{INTRODUCTION}

The sudden change of a parameter in the Hamiltonian (quantum quench) is the most common protocol to probe the quantum dynamics in isolated systems. ${ }^{1}$ This has stimulated the investigation of fundamental questions in quantum statistical mechanics, as e.g. the conditions under which a closed systems in an initial excited state is able or not to reach a thermal state. ${ }^{2-4}$ In various systems this has led to the observation of several intriguing dynamical behaviors, like long-time trapping in prethermal states ${ }^{5-8}$ or transitions between different dynamical regimes separated by dynamical critical points. ${ }^{9-13}$

The effects of a quantum quench can be ultimately related to a finite energy injection into the system, which simply results from the fact that the initial state $\left|\Psi_{i}\right\rangle$ is not the ground state of the Hamiltonian $\mathcal{H}_{f}$ setting the unitary time evolution. The opposite limit corresponds to an adiabatic process for which the energy injected into the system vanishes during the Hamiltonian variation.

In principle, an adiabatic process can be realized for an infinitely slow variation of some control parameter, leading to the continuous evolution of the equilibrium properties of the system from the initial to the final Hamiltonian. In practice, exceptions to this expectation are known. This is the case, for example, of gapless low-dimensional systems, where the energy density may increase with the system size preventing the adiabatic limit to be reached in the thermodynamic limit. ${ }^{14}$ Non-adiabatic dynamics may appear also for gapped low-dimensional systems in an ordered phase, where the reaching of the adiabatic limit is hampered by the vanishing critical temperature that leads to the melting of the ordered phase for every small, but finite, density of excitations. ${ }^{15}$ Recently, the formation of non-thermal states resulting from an adiabatic dynamics has been proposed in the case in which the initial state of the system is not described by a pure state. ${ }^{16}$

In the cases in which an adiabatic dynamics sets in, the time evolution following a finite rate variation of the Hamiltonian can be conveniently used to address the crossover between the quench and the equilibrium phase diagrams. In the presence of dynamical transitions, this has been used to highlight the connection between dynamical and equilibrium phase transition. In particular, the adiabatic mapping between dynamical and equilibrium critical points has been demonstrated in mean-field like models, as the $O(N)$ model in the large $N$ limit $^{17}$ and for the Mott transition in the repulsive Hubbard model in infinite dimension. ${ }^{18,19}$ This can be understood imaging an adiabatic dynamics which continuously explores the instantaneous evolution of the system ground state, so that a dynamical critical point surviving for a slow variation of the Hamiltonian is expected to merge with the quantum critical one in the adiabatic limit. It should be noticed that this mapping has not been so far addressed in models beyond mean-field. In addition, even in the cases in which the mapping holds, a departure from perfect adiabaticity is still expected for any finite speed crossing of a quantum critical point. ${ }^{20-25}$

In this paper we investigate the evolution from the quench to the adiabatic limit in the case of the attractive Hubbard model. The model gives the simplest description of the crossover between a weakly and a strongly coupled superconductor. ${ }^{26}$ Its dynamical properties are directly related to the investigation of the non-equilibrium properties of highly excited superconducting states which have recently attracted a considerable attention in relation to experiments in both solid state and cold atoms systems. ${ }^{27-30}$

Highly excited states induced by a sudden quench of the attractive interaction are characterized by the melting of the superconducting order at both weak and strong coupling. ${ }^{31-37}$ On the contrary, the superconducting properties of the ground state of the final Hamiltonian are expected to be recovered as the injected energy is reduced and the adiabatic limit is approached.

Here we use the time-dependent Gutzwiller approxi- 
mation ${ }^{38,39}$ to study how the ground state properties are recovered after a slow variation (finite ramp) of the attractive interaction. We show the existence of a weak and a strong coupling quench regimes for which two sharply different approaches to the adiabatic limit are observed. At weak coupling the recovery of the superconducting order depends only on the energy injected into the system. On the contrary, for quenches in the strong coupling limit the dynamics retains memory of the initial state and the recovery of the superconductivity does not depend only on the energy injected into the system.

The two dynamical regimes are closely related to a weak-to-strong coupling dynamical transition associated to different excitations created during the quench. By tracking the finite ramp evolution of the dynamical phase diagram and comparing to the dynamics in the nonsuperconducting phase we argue that the memory dependent dynamics in the strong coupling quench regime is due to the lack of an adiabatic connection between the non-equilibrium states at strong coupling and any equilibrium state at a finite value of the interaction.

In the following we will introduce the model and the method in Sec. II. The quench phase diagram obtained within the time-dependent Gutzwiller approach is discussed in Sec. III. Finally, Sec. IV reports the evolution of the quench phase diagram and the recovery of the adiabatic limit following a finite ramp dynamics.

\section{MODEL AND METHOD}

We study the attractive Hubbard model subject to a time-dependent interaction which is varied over a time $\tau$

$$
\mathcal{H}(t)=-\sum_{i j, \sigma} t_{i j} c_{i \sigma}^{\dagger} c_{j \sigma}-U_{\tau}(t) \sum_{i} n_{i \uparrow} n_{i \downarrow} .
$$

The attractive interaction is defined as

$$
U_{\tau}(t)=U_{i}+r_{\tau}(t)\left(U_{f}-U_{i}\right)
$$

where $U_{i}>0$ and $U_{f}>0$ are, respectively, the initial and final interaction and $r_{\tau}$ is a smooth ramping function interpolating between 0 and 1 over the time $\tau$. Its definition reads $r_{\tau}(t)=1 / 2-3 / 4 \cos \pi t / \tau+1 / 4 \cos ^{3} \pi t / \tau$ for $t<\tau$ and $r_{\tau}(t)=1$ for $t \geq \tau$. The chemical potential is chosen such that the system is half-filled with an average occupation of one electron per site $\langle n\rangle=1$.

The dynamics is studied using the time dependent Gutzwiller (TDG) variational ansatz ${ }^{38}$

$$
|\Psi(t)\rangle \equiv \prod_{i} \mathcal{P}_{i}(t)\left|\Psi_{0}(t)\right\rangle
$$

where $\left|\Psi_{0}(t)\right\rangle$ is a single particle wavefunction, describing the dynamics of the delocalized quasiparticles and $\mathcal{P}_{i}(t)$ are local projectors describing the dynamics of the local many body multiplets. The dynamics of $|\Psi(t)\rangle$ is obtained by means of the time dependent variational principle $\delta \int\left\langle\Psi(t)\left|i \partial_{t}-\mathcal{H}(t)\right| \Psi(t)\right\rangle=0$. The time-variational principle can be exactly imposed in the limit of a lattice with infinite coordination number $z$. In this paper we focus on this limit by considering a Bethe lattice with a semicircular density of states.

The imposition of the time dependent variational leads to a set of equations of motions which fully describes the dynamics of the system under a generic time-dependent perturbation. In few words, the dynamics is described by a set of coupled equations of motion for the delocalized quasiparticles, described by $\left|\Psi_{0}(t)\right\rangle$, and for the localized degrees of freedom, described by the projectors $\mathcal{P}$. The projectors $\mathcal{P}$ contain all the local variational parameter and are practically expressed in terms of matrices $\hat{\Phi}$ of the size of the local Hilbert space. ${ }^{38}$ Upon imposing the timedependent variational principle the following equations of motions are obtained

$$
\begin{aligned}
& i \partial_{t}\left|\Psi_{0}(t)\right\rangle=\mathcal{H}_{q p}[\hat{\Phi}]\left|\Psi_{0}(t)\right\rangle \\
& i \partial_{t} \hat{\Phi}(t)=\mathcal{H}_{l o c}(t) \hat{\Phi}(t)+\frac{\delta E_{k i n}(t)}{\delta \hat{\Phi}^{\dagger}(t)}
\end{aligned}
$$

$\mathcal{H}_{q p}$ is a BCS-like Hamiltonian which depends on the local variational parameters $\hat{\Phi}$ and $\mathcal{H}_{\text {loc }}(t)$ represents the local interaction terms in the Hamiltonian Eq. 1. $E_{k i n}(t)=\left\langle\Psi_{0}(t)\left|\mathcal{H}_{q p}[\hat{\Phi}]\right| \Psi_{0}(t)\right\rangle$ and $E_{p o t}(t)=$ $\operatorname{Tr}\left(\hat{\Phi}^{\dagger}(t) \mathcal{H}_{\text {loc }} \hat{\Phi}(t)\right)$ give, respectively, the kinetic and potential energies. The total energy $E(t)=E_{k i n}(t)+$ $E_{\text {pot }}(t)$ is conserved by the unitary dynamics (Eqs. 5-4) if the Hamiltonian does not depend on time. We refer to the literature for the detailed description of the method ${ }^{38}$ and its extension to the superconducting case. ${ }^{40}$

As can be appreciated from Eqs. 4-5, within the TDG the electron dynamics is described by the an effective single-particle problem (Eq. 4) which is self-consistently coupled to the dynamics of the local degrees of freedom (Eq. 5). This provides a mutual feedback between the delocalized and localized degrees of freedom which greatly improves the description of the non-equilibrium dynamics in correlated systems with respect to standard mean-field techniques. On the other hand, similarly to the standard mean-field methods, the effective single particle description of the many-body problem has an intrinsic limitation due to the lack of a finite life time of the quasi-particles. This prevents for example a correct description of the long time relaxation to true thermal states.

Despite such a limitation, the method provides a meaningful description of the dynamics in the prethermal regimes, with a good qualitative agreement with exact approaches in infinite dimensions such as the nonequilibrium Dynamical Mean Field Theory (DMFT). ${ }^{41}$ This can be appreciated by a direct comparison of the available results in the literature.

The simplest example is given by the quench dynamics in the paramagnetic repulsive Hubbard model ${ }^{9,10}$. In this case the variational ansatz Eq. 3 describes a dynamical transition as a function of the final interaction ${ }^{10}$ which correctly reproduces the trapping into quasi-stationary pre-thermal states observed by DMFT at both weak and 
strong coupling ${ }^{9}$ before thermalization eventually occurs on a longer time scale.

Another case is represented by the quench dynamics in the presence of long range ordered states in the antiferromagnetic (AFM) repulsive Hubbard model. ${ }^{34-37}$ This model is related to the Hamiltonian Eq. (1) by the particle-hole transformation $c_{i \uparrow}^{\dagger}=(-1)^{i} c_{i \uparrow}$, so that in the particle-hole symmetric case the two dynamics are equivalent. In this case both $\mathrm{DMFT}^{34-36}$ and $\mathrm{TDG}^{37}$ predict the trapping into non-thermal states for which a finite order parameter is observed despite the energy injected by the quench would predict a thermal melting of the initial long range order.

For later convenience we recall in the next section the results of the quench dynamics in the language of the superconducting order parameter for the present attractive Hubbard model. In the rest of the paper we consider a Bethe lattice in the limit $z \rightarrow \infty$ described by a semi-circular density of states of width $W, \rho(\epsilon)=$ $\theta(|W|-\epsilon) 4 / \pi \sqrt{W^{2}-4 \epsilon^{2}}$.

In the case of finite coordination lattices the use of the Gutzwiller approximation corresponds to the neglect of the spatial correlations. In the presence of a long-range order we expect that the spatial correlations play a minor role. Therefore, the following results can be expected to hold qualitatively for any lattice as long as it allows the establish of a long-range order at finite temperature.

We measure all the energies with respect to $W$ and all the times with respect to $W^{-1}$.

\section{SUDDEN QUENCH DYNAMICS}

At equilibrium the ground state of the Hamiltonian displays a finite superconducting order parameter for $U>0$. At half-filling the superconducting order is degenerate with a charge density wave instability. In the following we will constrain the dynamics to superconducting states and neglect possible competitions between the two orders. ${ }^{42}$

Fig. 1 summarizes the main results of the quench dynamics, corresponding to the limit $\tau \rightarrow 0$ in Eq. 2. Due to the effective single particle description of the electron dynamics any generic time dependent observable $\mathcal{O}(t)$ usually displays undamped oscillations around stationary values ${ }^{10}$ which are extracted by means of long-time averages

$$
\overline{\mathcal{O}}=\lim _{t \rightarrow \infty} \frac{1}{t} \int_{0}^{t} d \tau\langle\Psi(\tau)|\mathcal{O}| \Psi(\tau)\rangle .
$$

Panels (a) and (b) report the stationary values of the order parameter $\phi_{s c}=\left\langle c_{i \uparrow}^{\dagger} c_{i \downarrow}^{\dagger}\right\rangle$ as a function of $U_{f}$ and $U_{i}=0.25$ (a) and $U_{i}=1.0$ (b) compared to the zero temperature equilibrium value.

The stationary order parameter after the quench results always smaller than the corresponding ground state value of the final Hamiltonian as result of the fact that
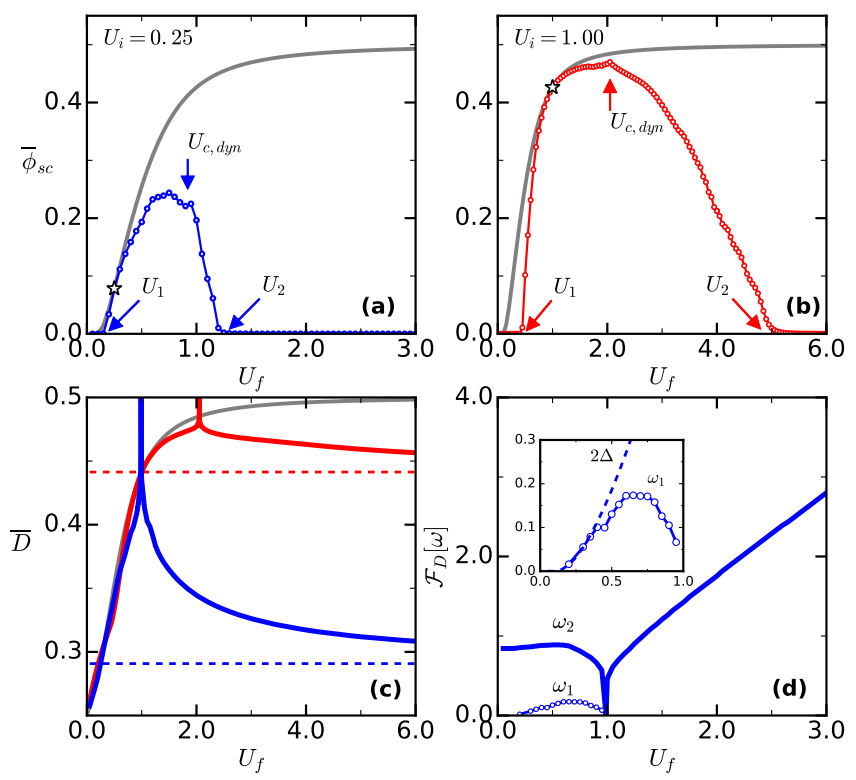

Figure 1. Sudden quench phase diagram. Top panels: (a)(b) stationary values of the superconducting order parameter after a sudden quench as a function of the final value of the interaction $U_{f}$ and for two initial values (indicated by stars) $U_{i}=0.25$ (a) and $U_{i}=1.0(\mathrm{~b})$. The solid line represents the equilibrium value of the order parameter corresponding to $U_{f}$. The arrows indicate the positions of the symmetry restoration critical points $U_{1}$ and $U_{2}$ and of the underlying weak-to-strong coupling dynamical transitions $U_{c, d y n}$ (see text). Bottom panels: (c) stationary values of the density of doubly occupied sites as a function of the final value of the interaction $U_{f}$ for $U_{i}=0.25$ (blue) and $U_{i}=1.0$ (red). Dashed lines indicate the initial values $D_{i}$. A sharp non-analyticity point appears at the dynamical transition as $D \rightarrow 1 / 2$. Grey line: equilibrium values corresponding to $U_{f}$. (d) Main peaks of the Fourier spectrum of the double occupancies $\mathcal{F}_{D}$ as a function of $U_{f}$. Only the case $U_{i}=0.25$ is shown. Inset: blow up of the $\omega_{1}$ frequency compared to the superconducting gap at equilibrium (dashed line). The two match in the limit $U_{f}=U_{i}$.

the quench corresponds to a finite injection of energy into the system. For final values of the interaction larger and smaller than the initial one, there exists two critical points depending on the value $U_{i}, U_{1}<U_{i}$ and $U_{2}>U_{i}$, for which the quench dynamics leads to the complete melting of the long range order.

The melting of the superconducting order can be understood by the excess of energy inserted into the system by the quench. This brings the system in some high temperature state for which the symmetry is restored. However, it has been shown that both critical points display non-thermal behaviors, ${ }^{34,37,43}$ meaning that the order parameter remains finite even for values $U_{f}$ for which an effective temperature larger than the equilibrium one is established.

Different origins for these non-thermal critical points have been discussed for the weak $\left(U_{1}\right)$ and strong $\left(U_{2}\right)$ coupling cases. At weak coupling a symmetry restoration 
due to dephasing has been invoked, ${ }^{34,37}$ while at strong coupling the stability of the symmetry broken state has been related to the long life-time of the excitations induced by the quench at large values of $U{ }^{36,43}$

The differences between quenches towards the weak and strong coupling limits are further highlighted by a sharp transition between two different dynamical regimes occurring for $U_{f}=U_{c, d y n}$ at intermediate values of the final interaction $U_{1}<U_{c, d y n}<U_{2}$. This can be appreciated in the dynamics of the double occupancies $D=\left\langle n_{i \uparrow} n_{i \downarrow}\right\rangle$ as a function of the final interaction. Looking at the stationary values $\bar{D}$ (panel (c)), we identify a weak coupling quench region $U_{f}<U_{c, d y n}$ in which $\bar{D}$ is renormalized according to the equilibrium expectation at the final interaction $\left(\bar{D}\right.$ increasing with $\left.U_{f}\right)$ and a strong coupling quench region in which the double occupancies decrease with $U_{f}$ getting more and more locked to their initial value with eventually $\bar{D} \rightarrow D_{i}$ for $U_{f} \rightarrow \infty$. For $U_{f}=U_{c, \text { dyn }}$, the dynamics of the double occupancies flows to the value $D=1 / 2$ which is a fixed point of the Gutzwiller dynamics. A sharp transition is therefore identified by a non-analyticity point in the $\bar{D}$ vs $U_{f}$ phase diagram (panel (c)).

Physical insight on the different dynamical regimes comes from the analysis of the frequency spectrum of the doublons dynamics $\mathcal{F}_{D}$ which gives the characteristic energies of the doublons excitations created during the quench. Fig. 1(d) reports the main peaks of $\mathcal{F}_{D}$ as a function of $U_{f}$. In the weak quench side $D$ oscillates with two main frequencies $\omega_{1}$ and $\omega_{2}$ which can be related to characteristic energy scales of the system. The larger frequency $\omega_{2}$ is of the order of the coherent quasi-particle bandwidth, whereas $\omega_{1}$ can be related to the non-equilibrium superconducting gap. This can be understood by looking at the inset of panel (d) where the frequency $\omega_{1}$ is compared to the equilibrium gap $2 \Delta . \omega_{1}$ follows the dome-like behaviour of the order parameter and merges with the equilibrium value $2 \Delta$ for $U_{f} \rightarrow U_{i}=0.25$. In the strong quench region $U_{f}>U_{c, \text { dyn }}$ only the mode $\omega_{2}$ survives and the frequency of the oscillations increases with $U_{f}$ as $\omega_{2} \sim U_{f}$.

We can give a physical interpretation of the excitations induced by the quench in the various regimes. In the weak coupling side these involve the low-energy coherent quasiparticle excitations, with the emergence of a collective amplitude mode $\left(\omega_{1}\right)$. On the other hand only high-energy excitations, approximatively located at the energies of the incoherent Hubbard bands, are activated for $U_{f}>U_{c, d y n}$. We can relate these excitations to local pair breaking processes that frustrate the growth of the double occupancies despite the large value of the final attractive interaction. The complete freezing of the excitations $(\omega \rightarrow 0)$ occurs at $U_{f}=U_{c, d y n}$.

For the repulsive AFM model the occurrence of such sharp crossover between two different dynamical regimes has been identified with a dynamical transition ${ }^{37}$ similar to that observed in the paramagnetic case. ${ }^{8-10}$ The presence of a dynamical transition can be related to the corresponding equilibrium properties of the model. In the paramagnetic case this has led to the identification of the dynamical transition as the dynamical counterpart of the Brinkmann-Rice metal-to-insulator transition. ${ }^{10}$ This has been further supported by the fact that the dynamical critical point merges to the equilibrium Mott transition for an adiabatically slow change of the interaction parameter. ${ }^{18,19}$

A similar analogy can be tried to be extended to the dynamics in the presence of long range ordered states. In the AFM case the possibility of an incoherent antiferromagnet has been proposed. ${ }^{37}$ However, in the AFM case and in the equivalent attractive superconducting case, this cannot be related to any equilibrium counterpart. Indeed, in the symmetry broken phases, for both attractive and repulsive cases, the order parameter is finite for each value of the interaction and no interaction driven transition is retrieved in the zero temperature equilibrium phase diagram. We mention that, contrary to the paramagnetic case, the possible presence of a sharp transition between two dynamical regimes in the presence of longrange order has not been addressed so far within DMFT. However, clearly distinct dynamical properties have been shown to hold for quenches at weak ${ }^{34}$ and strong ${ }^{43}$ coupling, implying that a crossover between the two regimes is likely to take place at intermediate quenches.

It should be noticed that the equivalence between the repulsive and attractive cases breaks down away from half-filling. In particular, in the repulsive case the dynamical transition turns into a crossover at any finite doping, ${ }^{10}$ as related to the disappearance of the Mott transition for $\langle n\rangle \neq 1$. On the contrary, the metal-pairing insulator transition in the attractive case survives at finite doping where, in infinite dimensions, it becomes of the first order. ${ }^{44}$ Similarly, the dynamical transition in the normal (non-superconducting) phase of the attractive case survives at finite doping. In this work we did not consider the study of the dynamical transition away from half-filling in the superconducting phase. However we expect that a similar dynamical transition may be retrieved for $\langle n\rangle \neq 1$.

\section{FINITE RAMP DYNAMICS}

The above dynamical properties show the emergence of stationary regimes in which the dynamical properties are not fully understood by a simple comparison of the quench and equilibrium phase diagrams. The main goal of the rest of the paper is to investigate this connection by looking at the evolution from the sudden quench to the adiabatic limit, where the phase diagrams in Figs. 1(a)(b) are expected to merge with the zero temperature equilibrium one (grey lines). In the following sections we will discuss such evolution by considering the variation of the interaction parameter over a finite time scale. 

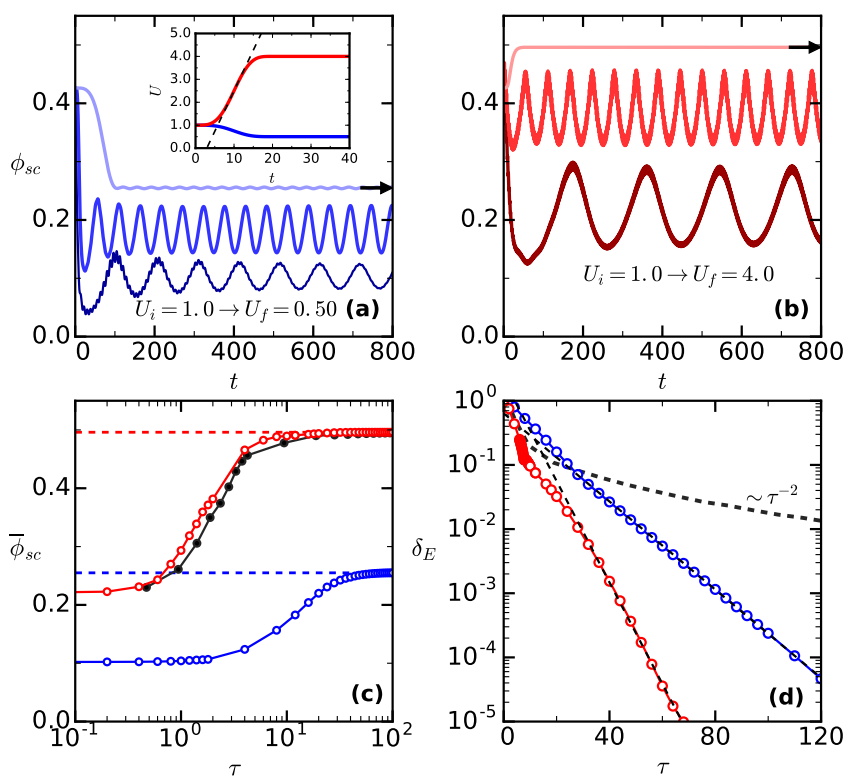

Figure 2. Adiabatic dynamics of superconducting states: Top panels (a)-(b): dynamics of the superconducting order parameter for an interaction change from $U_{i}=1.0$ to $U_{f}=0.5$ (a) and $U_{f}=4.0(\mathrm{~b})$ and different values of the ramping parameter. From bottom to top $\tau=0,12,120$ and $\tau=0,2,30$ for (a) and (b) panel respectively. The arrows indicate the ground state values for the final Hamiltonian. Inset: time dependent protocol for both values of $U_{f}$ and $\tau=20$. The dashed line indicates the linear approximation discussed in the text. Bottom panels: (c) stationary value of the order parameter as a function of the ramping time $\tau$ for $U_{f}=0.5$ (blue) and $U_{f}=4.0$ (red). Black dots represent the results for $U_{f}=4.0$ obtained with the linear approximation of the ramp. (d) Adiabaticity parameter (see text), color code as in (c). Red dashed line represents the adiabaticity parameter proportional to $\tau^{-2}$ for $U_{f}=4.0$ in the case of a linear ramp.

\section{A. Adiabatic dynamics of superconducting states}

We start considering the evolution of the nonequilibrium superconducting states for finite variation rates of the attractive interaction. Figs. 2(a)-(b) show the order parameter dynamics for two interaction quenches, respectively in the weak and strong coupling side of the quench phase diagram, $U_{i}=1.0$ to $U_{f}=0.5$ (a) and $U_{f}=4.0(\mathrm{~b})$, and different values of the ramping parameter $\tau$. Increasing the value of $\tau$ the order parameters become larger with respect to the sudden quench case. Eventually they reach the equilibrium value, showing that the dynamics approaches the adiabatic limit for slow enough variations of the interaction. The dynamics of the order parameter displays an undamped slow gap amplitude mode whose frequency increase as a function of $\tau$. This reflects the building up of a larger gap as the equilibrium ground state is approached in the adiabatic limit.

The time scale for the recovery of the adiabatic limit strongly depends on the final value of the interaction be- ing shorter for the strong coupling quench as compared to the weak coupling one. This can be appreciated in the stationary values of the order parameters as a function of $\tau$ (panel (c)). For the quench towards the weak coupling limit $\left(U_{f}=0.5\right)$ the equilibrium value is reached for $\tau \gtrsim 50$ whereas in the strong coupling case $\left(U_{f}=4.0\right)$ this limit is almost reached already for $\tau \gtrsim 15$.

The need for a shorter ramping time for the recovery of the equilibrium limit implies a smaller energy injection for fixed value of the ramping time. This fact can be understood on the basis of a Landau-Zener argument ${ }^{45,46}$ in relation to the gap of the final Hamiltonian. In the attractive Hubbard model the gap is a monotonically increasing function of the attractive interaction and for a larger the gap a smaller number of excitations is expected to be created during the continuous modification of the Hamiltonian at given variation rate.

We measure the energy injected during the ramp $\tau$ with respect to the sudden quench limit

$$
\delta_{E}(\tau)=\frac{E_{f}(\tau)-E_{e q}}{E_{0}-E_{e q}} .
$$

$E_{f}(\tau)=E_{f}(t=\tau)$ is the energy after the ramp which stays constant for $t>\tau$. $E_{0}=E_{f}(\tau=0)$ is the energy in the sudden quench limit and $E_{e q}$ is the zero temperature equilibrium energy corresponding to the final value of the interaction. For large ramping times $\delta_{E}$ decreases exponentially with $\tau$ on a scale that increases going from the weak to the strong coupling regime.

It is interesting to compare the above results with what obtained using a linear ramp. As can be seen in the inset of panel (a), $r_{\tau}(t)$ can be approximated in the middle of the ramp $t=\tau / 2$ with a line corresponding to $\tau^{\prime}=\frac{3}{4 \pi} \tau$. Comparing the stationary values of the order parameter we see that similar results are obtained for both protocols (full dots in panel (c)), namely the interpolation between the quench and the equilibrium values occurs for ramping times of the same order. On the other hand, the decay of the excitation energy changes for larger values of $\tau$. In particular, for the linear ramp an expected power law decay ${ }^{14} \delta_{E}(\tau) \sim \tau^{-2}$ is retrieved (dashed line in panel (d)). This means that at large $\tau$ the linear approximation of $r_{\tau}(t)$ becomes less and less accurate and the smooth variations of $r_{\tau}(t)$ for $t \simeq 0$ and $t \simeq \tau$ play a major role in determining the shorter ramping time needed to reach the adiabatic limit. Despite such a difference, the qualitative physics discussed in the rest of the paper does not depend on the ramping protocol.

\section{B. Adiabatic restoration of superconductivity}

We now investigate the adiabatic recovery of a finite order parameter for final values of the interaction for which the sudden quench dynamics leads to melting of the superconducting order. As done before, we start from the initial interaction $U_{i}=1.0$ and consider the ramp dynamics for $U_{f}=0.25$ and $U_{f}=6.0$, respectively in the 
weak $\left(U_{f}<U_{1}\right)$ and strong $\left(U_{f}>U_{2}\right)$ coupling regions of the sudden quench phase diagram (see Fig. 1(b)).

In Figs. 3(a)-(b) we show that increasing the ramping time the order parameter vanishes up to a critical value $\tau_{\star}$ above which superconductivity is recovered. For $\tau>\tau_{\star}$ the order parameter increases and eventually reaches the equilibrium value for $\tau \gg \tau_{\star}$. Similar features to that discussed in Fig. 2 are retrieved, namely the approach to the adiabatic limit is much slower in the weak coupling case compared to the strong coupling one.

The energy injected into the system and, in turn, the superconductivity restoration ramp time $\tau_{\star}$ depends on the initial state and its overlap with the excited states of the final Hamiltonian. Considering quenches to the same final Hamiltonian and changing the value of the initial interaction $U_{i}$ we observe that the superconductivity restoration ramp time $\tau_{\star}$ increases as $U_{i}$ is, respectively, increased for $U_{f}=0.25$ and decreased for $U_{f}=6.0$. This is expected by the fact that the larger is the difference $\Delta U=\left|U_{i}-U_{f}\right|$ the larger is the energy injected into the system and, for fixed final interaction, the slower is the variation rate needed to recover the adiabatic limit.

The relation between the injected energy during the ramp and the dynamical restoration of symmetry is further exploited in panels (c)-(d), where the stationary values of the order parameter obtained at each $\tau$ are plotted as a function of the final energy after the $\operatorname{ramp} E_{f}(\tau)$. Two clearly distinct behaviors emerge for quenches towards the weak and the strong coupling limits.

In the weak coupling case $\left(U_{f}=0.25\right)$ all the $\bar{\phi}\left(E_{f}\right)$ curves collapse onto a single one showing that the time evolved state approaches the steady state independently of the initial conditions for given value of the energy injected during the ramp. In particular, this reveals the existence of a universal value of the energy after the ramp $E_{f}=E_{\star} \simeq-0.1528$ above which the symmetry is restored.

A completely different scenario is obtained in the strong coupling case $\left(U_{f}=6.0\right)$ where different $\bar{\phi}_{s c}\left(E_{f}\right)$ curves are obtained for different values of the initial interaction. This shows that, contrary to the previous case, the dynamics retains memory of the initial state and, as a consequence, the energy threshold for the symmetry restoration is different for each value $U_{i}$.

We can try to extract from these results some information about the properties of the stationary dynamics and the mechanisms for the symmetry restoration. To do so we imagine to decompose the time evolving state for $t>\tau$ in terms of eigenstates of the final Hamiltonian

$$
|\Psi(t>\tau)\rangle=\sum_{n} c_{n} e^{-i \epsilon_{n} t}\left|\Psi_{n}^{f}\right\rangle
$$

where $\mathcal{H}_{f}\left|\Psi_{n}^{f}\right\rangle=\epsilon_{n}\left|\Psi_{n}^{f}\right\rangle$ and the coefficient $c_{n}$, such that $\sum_{n}\left|c_{n}\right|^{2}=1$, are determined by the dynamics during the ramp. Defining the expectation value of the the order parameter operator onto a single eigenstate $\phi_{n n},{ }^{47}$ the stationary value of the order parameter is described by
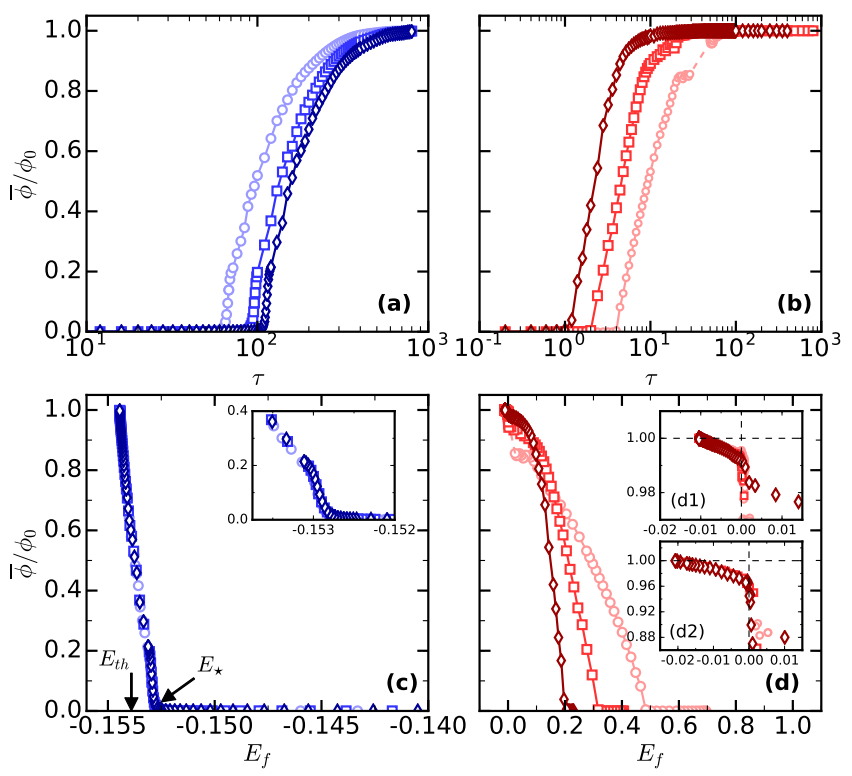

Figure 3. Adiabatic restoration of superconductivity. Top panels: stationary order parameters measured with respect to the equilibrium one at $U_{f}=0.25$ (a) and $U_{f}=6.0$ (b) as function of $\tau$ and different values of $U_{i}$. (a) $U_{i}=1.0$ (circles), $U_{i}=2.0$ (squares) and $U_{i}=3.0$ (diamonds). (b) $U_{i}=0.5$ (circles), $U_{i}=0.75$ (squares) and $U_{i}=1.0$ (diamonds). Bottom panels: stationary order parameters of panels (a)-(b) plotted as a function of the final energy after the ramp. Insets: (d1) Blow-up around $E_{f}=0$ of the data in panel (d). (d2) Same of $(\mathrm{d} 1)$ for a different set of $U_{i}$ and $U_{f}=3.0 . U_{i}=0.25$ (circles), $U_{i}=0.35$ (squares) and $U_{i}=0.45$ (diamonds).

the so called diagonal ensemble ${ }^{48}$

$$
\bar{\phi}=\sum_{n}\left|c_{n}\right|^{2} \phi_{n n} .
$$

In the weak coupling case the collapse onto a single curve of all the $\bar{\phi}\left(E_{f}\right)$ makes it reasonable to assume that the decomposition of the time evolved state is narrowly peaked in energy around the value of the final energy after the quench $E_{f}$ and that the expectation values smoothly varies in energy, as predicted by the Eigenstate Thermalization Hypothesis (ETH) ${ }^{2,3}$ and its extension to symmetry broken states. ${ }^{49}$ Under this assumption the existence of a universal energy $E_{\star}$ for the symmetry restoration shows, as originally proposed in Ref. 50, the existence of an energy edge in the spectrum of the final Hamiltonian which separates a subspace containing symmetry breaking eigenstates for $E<E_{\star}$ from a subspace containing only symmetry y invariant ones for $E>E_{\star}$. The dynamical restoration of symmetry for $E>E_{\star}$ is then due to the fact that the time evolving state overlaps only with symmetry invariant eigenstates so that the order parameter dephases to zero in the long time limit.

The dependence only on the energy $E_{f}$ may suggest a thermal origin for the restoration of symmetry. However, by comparing the energy threshold $E_{\star}$ with the internal energy corresponding to the equilibrium thermal transi- 
tion, $E_{t h} \equiv \operatorname{Tr}\left(e^{-\beta_{c} \mathcal{H}_{f}} \mathcal{H}_{f}\right) / \operatorname{Tr}\left(e^{-\beta_{c} \mathcal{H}_{f}}\right)$ with $\beta_{c}=1 / T_{c}$ the inverse of the critical temperature of the final Hamiltonian, we find that this is not the case being $E_{t h}<E_{\star}$ (see arrows in Fig. 3 (c)). In general, this reflects the fact that the Gutzwiller dynamics is not expected to describe the evolution towards a true thermal state. At the same time, we notice that the existence of an energy edge in the spectrum does not necessarily imply its equivalence with $E_{t h}$. Indeed, it may happen that the thermal transition occurs at $E_{t h}<E_{\star}$ due to the larger entropic contribution of the symmetry invariant states. ${ }^{50}$

The same analysis does not apply in the strong coupling regime where the memory of the initial state clearly highlights the breakdown of any thermal behavior. Two different scenarios can be considered. One possibility is that the ETH breaks down for the final Hamiltonian. In particular, the $c_{n}$ decomposition is peaked around the energy $E_{f}$ and the expectation values have large fluctuations for eigenstates close in energy, so that for different choices of $c_{n}$ different expectation values in the diagonal ensemble are obtained. On the other hand, if we assume ETH to hold we conclude that the decomposition of the time evolved wave function has to be widely spread in energy. In that case, assuming as before the existence of an energy edge $E_{\star}$ in the spectrum, for a given initial state a finite overlap with the symmetry broken states may be retained also for energies $E_{f}>E_{\star}$. Therefore the symmetry restoration can be understood as due to a spectral transfer from the low to the high energy part of the spectrum which strongly depends on the configuration of the initial state. While both are possible scenarios, a thorough analysis of the spectrum is clearly beyond the description of the present variational approach.

We observe that the strong dependence of the dynamics on the initial state might in principle suggest the absence of any adiabatic behavior. Indeed, if the stationary dynamics depends on both the energy pumped into the system and the initial state one could expect the convergence to different states in the long ramping time limit. Nonetheless, as seen in panel (b) the dynamics at strong coupling are all converging to the ground state of the final Hamiltonian, showing that the adiabatic limit is eventually recovered. In the following we investigate how the recovery of an adiabatic dynamics in the strong coupling case takes place for larger ramping times or, equivalently, smaller injected energies $E_{f}$.

\section{Recovery of the adiabatic limit and strong-to-weak coupling dynamical transition}

Inset (d1) in Fig. 3 shows the blow-up around the ground state energy of the final Hamiltonian $E_{f} \approx E_{e q}$ for the $\bar{\phi}\left(E_{f}\right)$ curves in panel (d). We observe that the adiabatic behavior is abruptly recovered as soon as the the energy crosses the value $E_{f}=0$, after which the collapse of all the different $\bar{\phi}_{s c}\left(E_{f}\right)$ curves onto a single one is retrieved. Choosing a different value of $U_{f}$ in
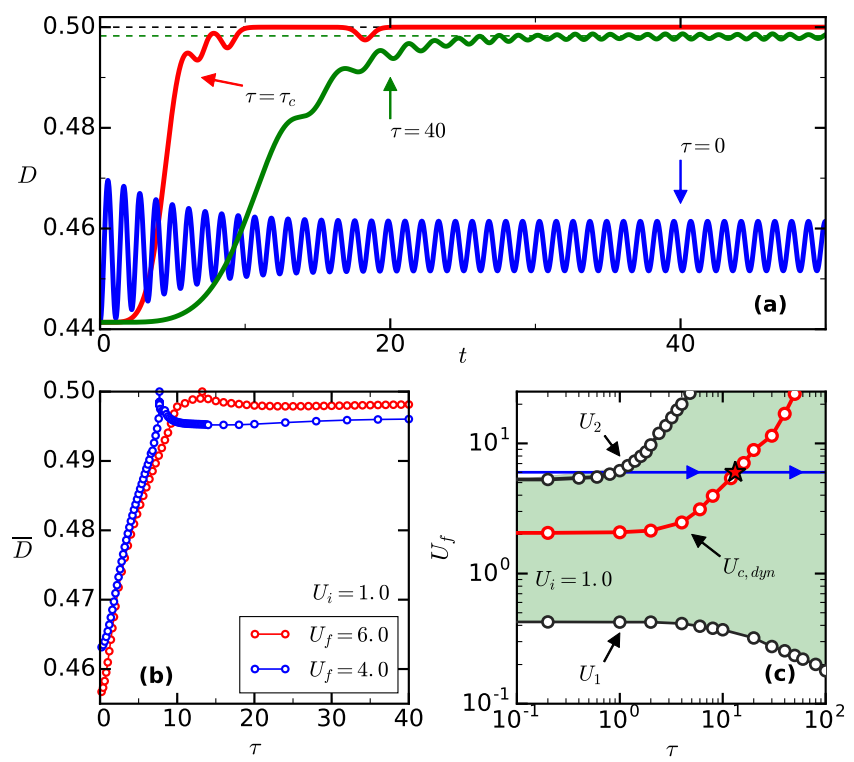

Figure 4. Dynamical transition as a function of $\tau$. (a): Dynamics of the double occupancies for $U_{i}=1.0 \rightarrow U_{f}=6.0$ and $\tau=0$ (blue line), $\tau=40$ (green line) and $\tau=13.2294$ (red line). Black and green dashed lines indicate respectively the values $D=1 / 2$ and the equilibrium value corresponding to $U_{f}$. (b): Stationary values of the double occupancies as a function of $\tau$ for $U_{i}=1.0 \rightarrow U_{f}=6.0$ (red) and $U_{i}=1.0 \rightarrow U_{f}=4.0$ (blue). (c): Dynamical phase diagram as a function of $\tau$. The symmetry restoration points $U_{1}$ and $U_{2}$ mark the boundaries in which a finite order parameter is observed (shaded area). Red points indicate the values of $U_{c, d y n}$ as a function of $\tau$. The horizontal arrows and the star indicate the crossing of the dynamical transition from the strong to the weak coupling region as a function of $\tau$ discussed in panels (a) and (b).

the strong quench regime and a different set of $U_{i}$ (inset (d2) in Fig. 3) we can see that the recovery of the collapse of the $\bar{\phi}_{s c}\left(E_{f}\right)$ for $E_{f}<0$ is a universal feature of quenches in the strong coupling regime. This fact highlights a clear link between the two different dynamical behaviors and the weak-to-strong quench dynamical transition discussed in Sec. III.

Within the Gutzwiller ansatz the energy $E=0$ is realized only for $D=1 / 2$ which, as already mentioned in Sec. III, is the fixed point of the dynamics defining the transition between the weak and the strong coupling quench dynamics. In Fig. 4 we show that such transition can be crossed as a function of the ramping time from the strong to the weak coupling side. In particular, in panel (a) we see that for the quench $U_{i}=1.0 \rightarrow U_{f}=6.0$ (corresponding to the diamonds in Fig. 3(d)) the fixed point of the dynamics is reached for the critical value $\tau_{c} \simeq 13.2294$, for which, after the value $D=1 / 2$ is reached, the dynamics stays constant apart from a small bump at $t \approx 18$ which is related to the numerical uncertainties in exactly reaching the fixed point.

In the quench limit $(\tau=0)$ the signatures of the strong 
coupling dynamics are visible, namely the double occupancies are locked around a value close to the initial one with fast oscillations of frequency $\sim U_{f}$. An almost adiabatic dynamics is established for $\tau=40$ where the double occupancies reach the equilibrium value with small residual oscillations. A non-analyticity point in the stationary value of the double occupancies is obtained for $\tau=\tau_{c}$ (panel (b)) which, similarly to what discussed in Sec. III, defines the crossing as a function of the ramping time $\tau$ of the dynamical transition from the strong $\left(\tau<\tau_{c}\right)$ and the weak $\left(\tau>\tau_{c}\right)$ coupling quench dynamical regimes. This corresponds respectively to $E_{f}\left(\tau<\tau_{c}\right)>0$ and $E_{f}\left(\tau>\tau_{c}\right)<0$, thus showing that the transition between the weak and the strong quench dynamics separates an adiabatic regime in which the dynamics depends only on the energy injected into the systems and a regime for which a dynamics strongly dependent on the initial state sets in.

In Fig. 4(c) we report the evolution of the dynamical phase diagram as a function of the ramping time. At fixed value of $\tau$ we define the two critical interactions $U_{1}(\tau)$ and $U_{2}(\tau)$ at which the symmetry is restored and the crossing of the dynamical transition $U_{c, d y n}(\tau)$ as the value of the final interaction at which the non-analyticity in the double occupancies occurs. In the adiabatic limit $(\tau \rightarrow \infty)$, where the quench phase diagram converges to the zero-temperature equilibrium one (see Fig. 1), we expect $U_{1}(\tau) \rightarrow 0$ and $U_{2}(\tau) \rightarrow \infty$. This is correctly reproduced in Fig. 4(c) where $U_{2}$ is an increasing function of $\tau$ and $U_{1}$ is slowly vanishing at large $\tau$.

At intermediate values $U_{c, d y n}(\tau)$ clearly follows the increase of $U_{2}$. Due to the absence of any interaction driven transition in the zero temperature equilibrium phase diagram we do not expect $U_{c, d y n}(\tau)$ to saturate at a finite value for $\tau \rightarrow \infty$. We thus conclude that also $U_{c, \text { dyn }}(\tau) \rightarrow \infty$ for $\tau \rightarrow \infty$, namely the strong coupling region disappears in the adiabatic limit and only the fixed point survives corresponding to the equilibrium atomic limit. As a consequence at fixed value of $U_{f}>U_{c, \text { dyn }}(\tau=0)$ the recovery of the adiabatic limit always implies the crossing of the strong-to-weak coupling quench dynamical transition (blue arrow in panel (c)). This shows that the non-equilibrium states at strong coupling lack of an adiabatic connection to the equilibrium ground state, meaning that they cannot be adiabatically transformed into the equilibrium ground state at finite $U$ without entering the weak coupling side of the transition.

\section{Comparison to the normal state dynamics}

The last result suggests a relation between the lack of an adiabatic connection to the equilibrium limit for the strong coupling case and the initial state dependent dynamics in Fig. 3(d). We check this by comparing the dynamics in the strong coupling regimes in both superconducting and normal (non-superconducting) phases for which an intrinsically different scenario is expected. In
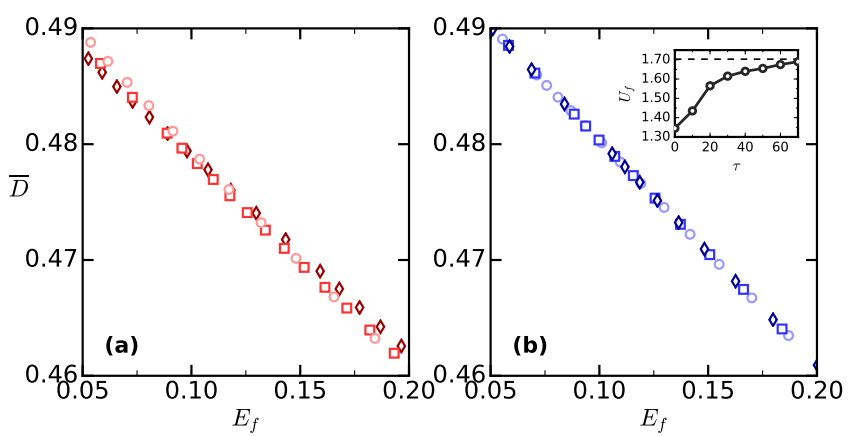

Figure 5. Stationary values of the double occupancies for the superconducting (a) and normal phases (b) as a function of the energy injected during the ramp for $U_{f}=6.0$ and different values of $U_{i} . U_{i}=1.0$ (circles), $U_{i}=0.75$ (squares) and $U_{i}=0.5$ (diamonds). Inset: $U_{c, d y n}$ as a function of $\tau$ for the normal phase. The dashed line indicates the equilibrium Mott transition.

the normal phase the weak-to-strong quench dynamical transition converges to the metal-pairing insulator Mott transition $U_{\text {Mott }}$ for $\tau \rightarrow \infty$ (see inset in Fig. 5(b)) and, contrary to the previous case, the non-equilibrium states for $U_{f}>U_{\text {Mott }}$ can be adiabatically transformed into the equilibrium pairing insulator without crossing any dynamical transition.

To compare the dynamics in the two phases we look at the dynamics of the double occupancies varying the ramping times and starting from different initial states. Fig. 5 reports the stationary values as a function of the energy after the ramp.

In the superconducting phase the same dependence on the initial state shown in Fig. 3 is reproduced. On the contrary the clear collapse onto a single curve is observed in the normal phase, for which a dynamics determined only by the final Hamiltonian is obtained also in the strong quench regime. This supports the fact that the initial state dependence in the superconducting phase is a manifestation of the lack of an adiabatic connection to the final ground state in the strong coupling quench regime.

\section{CONCLUSIONS}

We used the time-dependent Gutzwiller approximation to investigate the dynamics in the attractive Hubbard model. In particular, we focused on the connection between the sudden quench limit and the adiabatic dynamics following a slow variation of the attractive interaction.

The quench phase diagram displays two dynamical critical points at both weak and strong coupling at which the initial superconducting order parameter is melted after the quench and a sharp transition between a weak and a strong coupling quench dynamical regimes related to the interplay between the delocalized (quasiparticles) and the localized (Hubbard bands) degrees of freedom 
excited by the quench.

By changing the rate of variation of the attractive interaction we showed that the adiabatic limit is reached for sufficiently slow ramps. The ramping time for reaching the adiabatic limit depends on the final Hamiltonian and it decreases going from the weak to the strong coupling limit.

In the case of final values of the interaction for which the sudden quench dynamics leads to the complete melting of the superconducting order, we demonstrated the existence of a minimum ramping time for the recovery of a finite order parameter. For fixed final Hamiltonian the minimum ramping time depends on the initial state of the system and two distinct behaviors appear for quenches in the weak and strong coupling limit.

At weak coupling a dynamics depending only on the energy injected during the quench is realized independently of the initial state. On the contrary strong coupling quenches are characterized by a dynamics which retains memory of the initial state.

We showed that this is closely related to the weak-tostrong coupling quench dynamical transition which, for fixed final Hamiltonian, can be crossed as a function of the ramping time. In particular, the dynamics dependent on the initial state in the strong coupling side suddenly collapses onto the initial state independent dynamics as the transition to the weak coupling side is crossed.

By tracking the evolution of the dynamical phase diagram as a function of the ramping time and by a comparison to the dynamics in the normal phase we argued that the initial state dependent dynamics is the result of the lack of an adiabatic dynamics for the strong coupling quench regime in the superconducting phase.

\section{ACKNOWLEDGMENTS.}

I thank M. Schiró, M. Fabrizio and A. Georges for suggestions and insightful discussions. The research leading to these results has received funding from the European Research Council under the European Union's Seventh Framework Programme (FP7/2007-2013) / ERC Grant Agreement nr. 319286 (Q-MAC).
* giacomo.mazza@polytechnique.edu

1 Anatoli Polkovnikov, Krishnendu Sengupta, Alessandro Silva, and Mukund Vengalattore. Colloquium. Rev. Mod. Phys., 83:863-883, Aug 2011.

2 Mark Srednicki. Chaos and quantum thermalization. Phys. Rev. E, 50:888-901, Aug 1994.

3 Marcos Rigol, Vanja Dunjko, and Maxim Olshanii. Thermalization and its mechanism for generic isolated quantum systems. Nature, 452(7189):854-858, Apr 2008.

4 Adam M. Kaufman, M. Eric Tai, Alexander Lukin, Matthew Rispoli, Robert Schittko, Philipp M. Preiss, and Markus Greiner. Quantum thermalization through entanglement in an isolated many-body system. Science, 353(6301):794-800, 2016.

5 J. Berges, Sz. Borsányi, and C. Wetterich. Prethermalization. Phys. Rev. Lett., 93:142002, Sep 2004.

6 M. Gring, M. Kuhnert, T. Langen, T. Kitagawa, B. Rauer, M. Schreitl, I. Mazets, D. Adu Smith, E. Demler, and J. Schmiedmayer. Relaxation and prethermalization in an isolated quantum system. Science, 337(6100):1318-1322, 2012.

7 T. Langen, R. Geiger, M. Kuhnert, B. Rauer, and J. Schmiedmayer. Local emergence of thermal correlations in an isolated quantum many-body system. Nat Phys, 9(10):640-643, Oct 2013. Letter.

8 Michael Moeckel and Stefan Kehrein. Interaction quench in the Hubbard model. Phys. Rev. Lett., 100:175702, May 2008.

9 Martin Eckstein, Marcus Kollar, and Philipp Werner. Thermalization after an interaction quench in the Hubbard model. Phys. Rev. Lett., 103:056403, Jul 2009.

10 Marco Schiró and Michele Fabrizio. Time-Dependent Mean Field Theory for Quench Dynamics in Correlated Electron Systems. Phys. Rev. Lett., 105:076401, Aug 2010.
11 Bruno Sciolla and Giulio Biroli. Dynamical transitions and quantum quenches in mean-field models. Journal of Statistical Mechanics: Theory and Experiment, 2011(11):P11003, 2011.

12 Bruno Sciolla and Giulio Biroli. Quantum quenches and off-equilibrium dynamical transition in the infinitedimensional Bose-Hubbard model. Phys. Rev. Lett., 105:220401, Nov 2010.

13 A. Gambassi and P. Calabrese. Quantum quenches as classical critical films. EPL (Europhysics Letters), 95(6):66007, 2011.

14 Anatoli Polkovnikov and Vladimir Gritsev. Breakdown of the adiabatic limit in low-dimensional gapless systems. Nat Phys, 4(6):477-481, Jun 2008.

15 Anna Maraga, Pietro Smacchia, Michele Fabrizio, and Alessandro Silva. Nonadiabatic stationary behavior in a driven low-dimensional gapped system. Phys. Rev. B, 90(10), 2014.

16 D. M. Kennes. Adiabatically deformed ensemble: Engineering nonthermal states of matter. Phys. Rev. B, 96:024302, Jul 2017.

17 Anna Maraga, Pietro Smacchia, and Alessandro Silva. Linear ramps of the mass in the $O(N)$ model: Dynamical transition and quantum noise of excitations. Phys. Rev. B, 94(24):245122, 2016.

18 Matteo Sandri, Marco Schiró, and Michele Fabrizio. Linear ramps of interaction in the fermionic Hubbard model. Phys. Rev. B, 86, 2012.

19 Felix Hofmann, Martin Eckstein, and Michael Potthoff. Nonequilibrium self-energy functional approach to the dynamical Mott transition. Phys. Rev. B, 93:235104, Jun 2016.

${ }^{20}$ W. H. Zurek. Cosmological experiments in superfluid helium? Nature, 317(6037):505-508, 101985. 
${ }^{21} \mathrm{~T}$ W B Kibble. Topology of cosmic domains and strings. Journal of Physics A: Mathematical and General, 9(8):1387, 1976.

${ }^{22}$ Wojciech H. Zurek, Uwe Dorner, and Peter Zoller. Dynamics of a quantum phase transition. Phys. Rev. Lett., 95:105701, Sep 2005.

23 Jacek Dziarmaga. Dynamics of a quantum phase transition: Exact solution of the quantum Ising model. Phys. Rev. Lett., 95:245701, Dec 2005.

24 Anatoli Polkovnikov. Universal adiabatic dynamics in the vicinity of a quantum critical point. Phys. Rev. B, 72:161201, Oct 2005.

25 Anushya Chandran, Amir Erez, Steven S. Gubser, and S. L. Sondhi. Kibble-zurek problem: Universality and the scaling limit. Phys. Rev. B, 86:064304, Aug 2012.

${ }^{26}$ A Toschi, P Barone, M Capone, and C Castellani. Pairing and superconductivity from weak to strong coupling in the attractive Hubbard model. New Journal of Physics, 7(1):7, 2005.

27 D. Fausti, R. I. Tobey, N. Dean, S. Kaiser, A. Dienst, M. C. Hoffmann, S. Pyon, T. Takayama, H. Takagi, and A. Cavalleri. Light-induced superconductivity in a stripeordered cuprate. Science, 331(6014):189-191, 2011.

28 Daniel Greif, Thomas Uehlinger, Gregor Jotzu, Leticia Tarruell, and Tilman Esslinger. Short-range quantum magnetism of ultracold fermions in an optical lattice. Science, 340(6138):1307-1310, 2013.

29 Russell A. Hart, Pedro M. Duarte, Tsung-Lin Yang, Xinxing Liu, Thereza Paiva, Ehsan Khatami, Richard T. Scalettar, Nandini Trivedi, David A. Huse, and Randall G. Hulet. Observation of antiferromagnetic correlations in the Hubbard model with ultracold atoms. Nature, 519(7542):211214, Mar 2015. Letter.

30 A. Mazurenko, C. S. Chiu, G. Ji, M. Parsons, M. KanszNagy, R. Schmidt, F. Grusdt, E. Demler, D. Greif, and M. Greiner. A cold-atom Fermi-Hubbard antiferromagnet. Nature, 545(7655):462-466, May 2017. Letter.

31 R. A. Barankov and L. S. Levitov. Synchronization in the BCS pairing dynamics as a critical phenomenon. Phys. Rev. Lett., 96:230403, Jun 2006.

32 Emil A. Yuzbashyan and Maxim Dzero. Dynamical vanishing of the order parameter in a fermionic condensate. Phys. Rev. Lett., 96:230404, Jun 2006.

33 Francesco Peronaci, Marco Schiró, and Massimo Capone. Transient dynamics of $d$-wave superconductors after a sudden excitation. Phys. Rev. Lett., 115:257001, Dec 2015.

34 Naoto Tsuji, Martin Eckstein, and Philipp Werner. Nonthermal antiferromagnetic order and nonequilibrium criticality in the hubbard model. Phys. Rev. Lett., 110:136404, Mar 2013.

35 Philipp Werner, Naoto Tsuji, and Martin Eckstein. Nonthermal symmetry-broken states in the strongly interacting
Hubbard model. Phys. Rev. B, 86, 2051.

${ }^{36}$ Karsten Balzer, F. Alexander Wolf, Ian P. McCulloch, Philipp Werner, and Martin Eckstein. Nonthermal melting of néel order in the hubbard model. Phys. Rev. X, 5:031039, Sep 2015.

37 Matteo Sandri and Michele Fabrizio. Nonequilibrium dynamics in the antiferromagnetic Hubbard model. Phys. Rev. B, 88:165113, Oct 2013.

38 Michele Fabrizio. The Out-of-Equilibrium TimeDependent Gutzwiller Approximation. In Veljko Zlatic and Alex Hewson, editors, New Materials for Thermoelectric Applications: Theory and Experiment, pages 247-273. Springer Netherlands, 2013.

39 G. Seibold and J. Lorenzana. Time-dependent gutzwiller approximation for the hubbard model. Phys. Rev. Lett., 86:2605-2608, Mar 2001.

40 Giacomo Mazza and Antoine Georges. Nonequilibrium superconductivity in driven alkali-doped fullerides. Phys. Rev. B, 96:064515, Aug 2017.

41 Hideo Aoki, Naoto Tsuji, Martin Eckstein, Marcus Kollar, Takashi Oka, and Philipp Werner. Nonequilibrium dynamical mean-field theory and its applications. Rev. Mod. Phys., 86:779-837, Jun 2014.

42 M. A. Sentef, A. Tokuno, A. Georges, and C. Kollath. Theory of laser-controlled competing superconducting and charge orders. Phys. Rev. Lett., 118:087002, Feb 2017.

43 Philipp Werner, Naoto Tsuji, and Martin Eckstein. Nonthermal symmetry-broken states in the strongly interacting Hubbard model. Phys. Rev. B, 86:205101, Nov 2012.

44 M. Capone, C. Castellani, and M. Grilli. First-order pairing transition and single-particle spectral function in the attractive Hubbard model. Phys. Rev. Lett., 88:126403, Mar 2002.

45 L. D. Landau. To the theory of energy transmission in collissions. II. Phys. Zs. Sowjet., 2, p. 46, 1932 (English and German), 2:46, December 1932.

46 C. Zener. Non-Adiabatic Crossing of Energy Levels. Proceedings of the Royal Society of London Series A, 137:696702, September 1932.

47 The expectation value of the order parameter operator between two eigenstates is strictly zero $\phi_{n m}=$ $\left\langle\Psi_{n}\left|c_{i \uparrow}^{\dagger} c_{i \downarrow}^{\dagger}\right| \Psi_{m}\right\rangle=0$ for symmetry. The proper definition in this case should take into account the long range order $\left|\phi_{n m}\right|^{2}=\lim _{|i-j| \rightarrow \infty}\left\langle\Psi_{n}\left|c_{i \uparrow}^{\dagger} c_{i \downarrow}^{\dagger} c_{j \downarrow} c_{j \uparrow}\right| \Psi_{m}\right\rangle$.

48 We assume here that there are no degeneracy in the spectrum.

49 Keith R. Fratus and Mark Srednicki. Eigenstate thermalization in systems with spontaneously broken symmetry. Phys. Rev. E, 92:040103, Oct 2015.

50 Giacomo Mazza and Michele Fabrizio. Dynamical quantum phase transitions and broken-symmetry edges in the many-body eigenvalue spectrum. Phys. Rev. B, 86:184303, Nov 2012. 